\title{
PRIME IDEALS IN ALGEBRAS OF CONTINUOUS FUNCTIONS
}

\author{
H. G. DALES AND R. J. LOY
}

\begin{abstract}
Let $X_{0}$ be a compact Hausdorff space, and let $\mathbf{C}\left(X_{0}\right)$ be the Banach algebra of all continuous complex-valued functions on $X_{0}$. It is known that, assuming the continuum hypothesis, any nonmaximal, prime ideal $\mathbf{P}$ such that $\left|\mathbf{C}\left(X_{0}\right) / \mathbf{P}\right|=2^{\aleph_{0}}$ is the kernel of a discontinuous homomorphism from $\mathbf{C}\left(X_{0}\right)$ into some Banach algebra. Here we consider the converse question of which ideals can be the kernels of such a homomorphism. Partial results are obtained in the case where $X_{0}$ is metrizable.
\end{abstract}

Introduction. Let $X_{0}$ be a compact Hausdorff space, let $\mathbf{C}\left(X_{0}\right)$ be the Banach algebra of all continuous complex-valued functions on $X_{0}$, and let $\Theta$ be a homomorphism from $\mathbf{C}\left(X_{0}\right)$ into a Banach algebra B. It is an old question whether or not each such homomorphism is automatically continuous: we know that, if the continuum hypothesis $(\mathrm{CH})$ be assumed, there are discontinuous homomorphisms from C $\left(X_{0}\right)$ for each infinite space $X_{0}$ (see $\left.[2,8]\right)$, and also that there are models of set theory in which each such homomorphism is continuous $[15,4]$. Nevertheless, there remain a number of open questions about these homomorphisms. Some, involving the case where $\mathrm{CH}$ does not hold, are discussed in [4, Chapter 3]. Here, we consider a question about the kernels of discontinuous homomorphisms when $\mathrm{CH}$ does hold.

The seminal study of homomorphisms $\Theta: \mathbf{C}\left(X_{0}\right) \rightarrow \mathbf{B}$ is that of Bade and Curtis [1]. They showed, in particular, that, if $\Theta$ is discontinuous, there is a finite, nonempty set $F=\left\{x_{1}, \ldots, x_{n}\right\}$ of nonisolated points of $X_{0}$, a continuous homomorphism $\mu: \mathbf{C}\left(X_{0}\right) \rightarrow \mathbf{B}$, and linear maps $\lambda_{1}, \ldots, \lambda_{k}: \mathbf{C}\left(X_{0}\right) \rightarrow \mathbf{B}$, such that $B=\mu$ $+\lambda_{1}+\cdots+\lambda_{k}$, such that $\lambda_{i} \mid \mathbf{M}_{x_{i}}$ is a nonzero homomorphism into the radical of $\mathbf{B}$, and such that $\lambda_{i} \mid \mathbf{J}_{x_{i}}=0(i=1, \ldots, k)$. Here, $\mathbf{M}_{x}=\{f: f(x)=0\}$ and $\mathbf{J}_{x}=\{f$ : $f^{-1}(\{0\})$ is a neighborhood of $\left.x\right\}$ for $x \in X_{0}$. Since $\mathbf{J}_{x_{i}}$ is dense in $\mathbf{M}_{x_{i}}, \lambda_{i}$ is discontinuous $(i=1, \ldots, k)$. The set $F$ is the singularity set of $\Theta$.

Let $\Theta$ be a homomorphism from $\mathbf{C}\left(X_{0}\right)$. Then $q: f \mapsto\|\Theta(f)\|$ is a seminorm on $\mathbf{C}\left(X_{0}\right)$, and $q$ is continuous if and only if $\Theta$ is continuous. Let $\mathbf{F}_{q}$ be the set of nonmaximal, prime, $q$-closed ideals in $\mathbf{C}\left(X_{0}\right)$. It is shown by Esterle in [5] that $\mathbf{F}_{q}=\varnothing$ if and only if $q$ is discontinuous (see also [13]). In this case, set $\mathbf{I}_{q}=\bigcap\{\mathbf{P}$ : $\mathbf{P} \in \mathbf{F}_{q}$ \}. Then: (i) the set of maximal ideals of $\mathbf{C}\left(X_{0}\right)$ containing an element of $\mathbf{F}_{q}$ is finite; (ii) $\Theta \mid \mathbf{I}_{q}$ is continuous, and, if $\mathbf{I}$ is an ideal of $\mathbf{C}\left(X_{0}\right)$ such that $\Theta \mid \mathbf{I}$ is continuous, then $\mathbf{I} \subset \mathbf{I}_{q}$; (iii) each chain in $\mathbf{F}_{q}$ is well-ordered with respect to inclusion. This theorem of Esterle extends the earlier result of Bade and Curtis.

Received by the editors October 18, 1985.

1980 Mathematics Subject Classification (1985 Revision). Primary 46J10, 54C40.

Key words and phrases. Prime ideals, discontinuous homomorphisms.

(C)1986 American Mathematical Society $0002-9939 / 86 \$ 1.00+\$ .25$ per page 
It is hoped that $\mathbf{F}_{q}$ is a finite union of well-ordered chains. Clearly, this is so if and only if there exist $\mathbf{P}_{1}, \ldots, \mathbf{P}_{k} \in \mathbf{F}_{q}$ such that $\mathbf{I}_{q}=\mathbf{P}_{1} \cap \cdots \cap \mathbf{P}_{k}$. Again, slightly reformulating this hope, we are led to the following question [3, Question 15].

Question. Let $\Theta$ be a homomorphism from $\mathbf{C}\left(X_{0}\right)$ into a Banach algebra. Do there exist prime ideals $\mathbf{P}_{1}, \ldots, \mathbf{P}_{k}$ in $\mathbf{C}\left(X_{0}\right)$ such that $\Theta \mid \mathbf{P}_{1} \cap \cdots \cap \mathbf{P}_{k}$ is continuous?

The strongest form of the theorem asserting the existence of discontinuous homomorphisms from $\mathbf{C}\left(X_{0}\right)$ is the following [9]. We denote the cardinality of a set $S$ by $|S|$. Let $\mathbf{P}$ be a nonmaximal, prime ideal of $\mathbf{C}\left(X_{0}\right)$ such that $\left|\mathbf{C}\left(X_{0}\right) / \mathbf{P}\right|=\boldsymbol{\aleph}_{1}$. Then there exists a homomorphism from $\mathbf{C}\left(X_{0}\right)$ into a Banach algebra such that $\operatorname{ker} \Theta=\mathbf{P}$. If $X_{0}$ is infinite, then $\mathbf{C}\left(X_{0}\right)$ contains nonmaximal, prime ideals $\mathbf{P}$ such that $\left|\mathbf{C}\left(X_{0}\right) / \mathbf{P}\right|=2^{\aleph_{0}}$, and so, if $\mathrm{CH}$ holds, there are discontinuous homomorphisms from $\mathbf{C}\left(X_{0}\right)$. Further, if $\left|\mathbf{C}\left(X_{0}\right)\right|=2^{\aleph_{0}}$ and if $\mathbf{C H}$ holds, then each ideal of $\mathbf{C}\left(X_{0}\right)$ which is a finite intersection of nonmaximal, prime ideals is the kernel of such a homomorphism. Thus, a positive answer to the question would give the complete story about the domains of continuity of homomorphisms from $\mathbf{C}\left(X_{0}\right)$, at least if $\left|\mathrm{C}\left(X_{0}\right)\right|=2^{\aleph_{0}}$. (Incidently, we do not know whether or not every infinite-dimensional, nonnilpotent commutative Banach algebra $\mathbf{A}$ contains a nonmaximal, prime ideal $\mathbf{P}$ such that $|\mathbf{A} / \mathbf{P}|=2^{\boldsymbol{N}_{0}}$. If $\mathbf{A}$ is separable and an integral domain, then $\{0\}$ is certainly such an ideal, and it may be the only one [11].)

In attempting to answer our question, we reformulate it again by using a result of Johnson [12]. First, some notation that will be fixed for the remainder of this note. Let $X$ be a locally compact space, let $\mathbf{C}_{0}(X)$ be the space of continuous functions on $X$ which vanish at infinity, and denote by $\beta X$ the Stone-Čech compactification of $X$. For $f \in \mathbf{C}_{0}(X)$, let $\hat{f}$ be the continuous extension of $f$ to $\beta X$. For $p \in \beta X \backslash X$, set

$$
\mathbf{J}_{p}=\left\{f \in \mathbf{C}_{0}(X): \hat{f}^{-1}(\{0\}) \text { is a neighborhood of } p \text { in } \beta X\right\} .
$$

Johnson's theorem, which extends that of Bade and Curtis, is the following. Let $X_{0}$ be a compact space, let $\Theta: \mathbf{C}\left(X_{0}\right) \rightarrow \mathbf{B}$ be a discontinuous homomorphism with singularity set $F$, and let $X=X_{0} \backslash F$. Identify $\mathbf{C}_{0}(X)$ with $\left\{f \in \mathbf{C}\left(X_{0}\right): f \mid F=0\right\}$. Then there exist $p_{1}, \ldots, p_{n} \in \beta X \backslash X$, a continuous homomorphism $\mu: \mathbf{C}\left(X_{0}\right) \rightarrow \mathbf{B}$, and linear maps $\nu_{1}, \ldots, \nu_{n}: \mathbf{C}\left(X_{0}\right) \rightarrow \mathbf{B}$ such that $\Theta=\mu+\nu_{1}+\cdots+\nu_{n}$ and $\nu_{i} \mid \mathbf{C}_{0}(X)$ is a homomorphism with $\nu_{i} \mid \mathbf{J}_{p}=0(i=1, \ldots, n)$. Necessarily, the range of each $\nu_{i}$ is contained in the radical of $\mathbf{B}$. A direct proof of this result is given in $[4$, Chapter 1].

Thus the question we raised is equivalent to the following. Let $X$ be a locally compact space, let $p \in \beta X \backslash X$, and let $\nu: \mathbf{C}_{0}(X) \rightarrow \mathbf{R}$ be a homomorphism from $\mathbf{C}_{0}(X)$ into a radical Banach algebra $\mathbf{R}$ such that $\operatorname{ker} \nu \supset \mathbf{J}_{p}$. Is $\operatorname{ker} \nu$ a finite intersection of prime ideals?

The purpose of the present note is to make a modest start towards this problem by analysing when we can have $\operatorname{ker} \nu$ equal to $\mathbf{J}_{p}$ in the above situation, for suitably restricted $X$. We also determine when $\mathbf{J}_{p}$ is itself a prime ideal. 
Results. Let $X$ be a locally compact space. For $S \subset X$, denote by $\bar{S}$ the closure of $S$ in $\beta X$. If $U$ is open in $\beta X$, then $\overline{U \cap X}=\bar{U}$. If $S$ and $T$ are zero-sets of continuous functions on $X$, then $\bar{S} \cap \bar{T}=\overline{S \cap T}$; in particular, if $X$ is metrizable, this holds for any closed sets $S$ and $T$ in $X$. See [10, Chapter 6] for the elementary properties of $\beta X$. Denote by $W$ the (open) subset of $X$ consisting of isolated points, and by $K$ the (closed) subset of $X$ consisting of nonisolated points. Define

$$
\begin{array}{r}
P=(\bar{W} \backslash \bar{K}) \cup\{p \in \bar{K} \backslash \bar{W}: p \text { is not a limit point in } \beta X \\
\text { of a discrete subset of } K \backslash \bar{W}\} .
\end{array}
$$

THEOREM. Let $X$ be a locally compact, $\sigma$-compact, metrizable space, and suppose that $C H$ holds. Then for a point $p \in \beta X \backslash X$ the following are equivalent:

(1) $p \in P$;

(2) $\mathbf{J}_{p}$ is a prime ideal;

(3) $\mathbf{J}_{p}$ is a finite intersection of prime ideals;

(4) $\mathbf{J}_{p}$ is the kernel of a discontinuous homomorphism from $\mathbf{C}_{0}(X)$ into a (radical) Banach algebra.

Proof. Since $\beta X=\bar{K} \cup \bar{W}$, the point $p \in \beta X$ lies in exactly one of $\bar{K} \cap \bar{W}$, $\bar{W} \backslash \bar{K}$, and $\bar{K} \backslash \bar{W}$; we consider the three possibilities in turn. Let $d$ denote the metric on $X$, and, for $x \in X$ and $\varepsilon>0$, let $B(x, \varepsilon)=\{y \in X: d(x, y)<\varepsilon\}$.

Suppose first that $p \in \bar{K} \cap \bar{W}$, so that $p \notin P$. Note that

$$
\bar{K} \cap \bar{W}=(K \cap \bar{W} \cap X)^{-}=(K \cap \bar{W})^{-}
$$

since both $K$ and $\bar{W} \cap X$ are closed in $X$. Since $X$ is $\sigma$-compact, we can write $K \cap \bar{W}=\bigcup E_{n}$, where each $E_{n}$ is a compact subset of $X$, and $E_{n} \subset E_{n+1}$ (strictly) $(n=1,2, \ldots)$. Noting that $\bigcup E_{n} \subset \bar{W} \backslash W$, inductively construct finite subsets $W_{n}$ of $W$, and a sequence $\left(\alpha_{n}\right)$ of positive reals as follows. Choose $x \in W$, set $\alpha_{1}=1$, $W_{1}=\{x\}$. Given $W_{1}, \ldots, W_{n}$, set $\delta_{n}=d\left(E_{n+1}, W_{1} \cup \cdots \cup W_{n}\right)$, and take $0<\alpha_{n+1}$ $<\min \left(\frac{1}{2} \delta_{n}, \alpha_{n}, n^{-1}\right)$. Take a finite cover of $E_{n+1}$ by open balls of radius $\alpha_{n+1}$, and choose one point of $W$ in each ball to form the set $W_{n+1}$. By the construction $W_{i} \cap W_{j}=\varnothing$ if $i \neq j$.

Let $\left\{\sigma_{m}: m \in \mathbf{N}\right\}$ be an infinite partition of $\mathbf{N}$ into infinite sets, and let $A_{m}=\bigcup\left\{W_{n}: n \in \sigma_{m}\right\}$. Let $f_{n}=1 / n$ on the set $W_{n}$ and 0 elsewhere. Since $W_{n}$ is a finite set of isolated points, $f_{n} \in \mathbf{C}_{0}(X)$. Set $g_{m}=\Sigma\left\{f_{n}: n \in \sigma_{m}\right\}$, so that $g_{m} \in$ $\mathrm{C}_{0}(X)(m=1,2, \ldots)$.

Now take a neighborhood $U$ of $p$ in $\beta X$. Since $p \in(K \cap \bar{W})^{-}$, there is a point $x \in K \cap \bar{W} \cap U$; take $\varepsilon>0$ so that $B(x, \varepsilon) \subset U$ and choose $k$ such that $\alpha_{k}<\frac{1}{2} \varepsilon$. Since $\sigma_{m}$ is infinite, there is an $n \in \sigma_{m}$ with $n>k$. But then $W_{n} \cap B(x, \varepsilon) \neq \varnothing$, hence $A_{m} \cap U \neq \varnothing$, and so $g_{m} \notin \mathbf{J}_{p}$. On the other hand, $g_{m} g_{n}=0$ for $m \neq n$ because $A_{m} \cap A_{n}=\varnothing$, and so [1, Theorem 5.3] shows that $\mathbf{J}_{p}$ cannot be the kernel of a radical homomorphism.

Secondly, consider the case where $p \in \bar{W} \backslash \bar{K}$, so that $p \in P$. Take $f, g \in \mathbf{C}_{0}(X)$ with $f g \in \mathbf{J}_{p}$. Let $U$ be a neighborhood of $p$ in $\beta X$ with $\bar{U} \cap \bar{K}=\varnothing$, and set

$$
\begin{aligned}
& V_{1}=\{x \in \bar{U} \cap X:|f(x)|<|g(x)|\}, \\
& V_{2}=\{x \in \bar{U} \cap X:|f(x)| \geqslant|g(x)|\} .
\end{aligned}
$$


Since $V_{1}, V_{2} \subset W$, both these sets are open in $X$. But $V_{1} \cup V_{2}=\bar{U} \cup X$, which is closed in $X$. Thus both $V_{1}$ and $V_{2}$ are clopen in $X$. Hence $\bar{V}_{1} \cap \bar{V}_{2}=\bar{V}_{1} \cap V_{2}=\varnothing$, and so exactly one of $\bar{V}_{1}$ and $\bar{V}_{2}$ is a neighborhood of $p$. Since on a possibly smaller neighborhood $f g$ vanishes, it follows that one of $f$ and $g$ lies in $\mathbf{J}_{p}$, and hence that $\mathbf{J}_{p}$ is prime.

Thirdly, suppose that $p \in \bar{K} \backslash \bar{W}$, and take the case that $p$ is a limit point of a discrete subset $D$ of $K \backslash \bar{W}$, so that $p \notin P$. Since $X$ is second countable, $D$ must be countable, say $D=\left\{x_{n}\right\}$. Let $\left\{U_{n}\right\}$ be a collection of open sets in $X$ with $x_{n} \in U_{n}$ and $U_{n} \cap U_{m}=\varnothing(n, m \in \mathbf{N}, n \neq m)$. Further, since each $x_{n}$ is nonisolated in $X$, there exist $\left\{x_{m}^{n}\right\} \subset U_{n} \backslash\left\{x_{n}\right\}$ with $x_{m}^{n} \rightarrow x_{n}$ as $m \rightarrow \infty$, and $x_{r}^{n} \neq x_{s}^{n}$ if $r \neq s$. Now choose open sets $V_{m}^{n} \subset U_{n}$ with $x_{m}^{n} \in V_{m}^{n}$ and $V_{r}^{n} \cap V_{s}^{n}=\varnothing$ if $r \neq s$. Finally, choose continuous, nonnegative real-valued functions $\left\{f_{n}\right\}$ on $X_{0}$ such that

(a) $\operatorname{supp}\left(f_{m}\right) \subset \bigcup\left\{V_{m}^{n}: n \in \mathbf{N}\right\}$;

(b) $f_{m}\left(x_{m}^{n}\right)=(n+m)^{-1}=\sup \left\{f_{m}(x): x \in V_{m}^{n}\right\}$.

Let $\left\{\sigma_{m}: m \in \mathbf{N}\right\}$ be an infinite partition of $\mathbf{N}$ into infinite sets and let

$$
F_{m}=\sum\left\{f_{n}: n \in \sigma_{m}\right\} \text {. }
$$

The $F_{m}$ are well defined since the summands have disjoint support, and lie in $\mathrm{C}_{0}(X)$ by (b). If $Z$ is a neighborhood of $p$ in $\beta X$, then $x_{n} \in Z$ for some $n$, whence for each $i, x_{j}^{n} \in Z$ for $j \in \sigma_{i}$ with $j$ sufficiently large. Thus $F_{i}$ is not identically zero on $Z \cap X$, that is, $F_{i} \notin \mathbf{J}_{p}$. But $F_{i} F_{j}=0$ if $i \neq j$, and so, as above, $\mathbf{J}_{p}$ cannot be the kernel of a discontinuous homomorphism.

There remains the case where $p \in \bar{K} \backslash \bar{W}$, and $p$ is not a limit point of a discrete subset of $K \backslash \bar{W}$. Here we show that $\mathbf{J}_{p}$ is again prime. Suppose then that $f$ and $g \in \mathbf{C}_{0}(X)$ with $f g \in \mathbf{J}_{p}$. Let

$$
\begin{gathered}
G_{1}=\{x \in X:|f(x)| \leqslant|g(x)|\}, \\
G_{2}=\{x \in X:|f(x)| \geqslant|g(x)|\}, \\
G=\{x \in X:|f(x)|=|g(x)|\},
\end{gathered}
$$

so that $G=G_{1} \cap G_{2}$, As above, if $p \notin \bar{G}_{1}$ or $p \notin \bar{G}_{2}$, then one of $f$ or $g$ lies in $\mathbf{J}_{p}$. Thus we may suppose that $p \in \bar{G}$, that is that $p \in(\bar{G} \cap \bar{K}) \backslash \bar{W}=\overline{G \cap K} \backslash \bar{W}$.

Let $Y=$ int $_{K}(G \cap K)$ and $Z=\partial_{K}(G \cap K)$, the frontier of $G \cap K$ in $K$. Since $Z=\partial_{K} R$, where $R=\{x \in K:|f(x)| \neq|g(x)|\}$, an open set in $K$, it follows from a theorem of Hausdorff [14, Lemma 4.39] that $Z$ is the set of limit points (in $K$ ) of a discrete subset $D$ of $K$. But then $\bar{Z} \subset \bar{D}$, and so by hypothesis $p \notin \bar{Z}$. Let $U$ be a neighborhood of $p$ in $\beta X$ such that $\bar{U} \cap(\bar{Z} \cup \bar{W})=\varnothing$ and $\hat{f} \hat{g} \mid U=0$, and set $K_{1}=\bar{U} \cap G, K_{2}=\bar{U} \cap(K \backslash Y)$. Then $K_{1}$ and $K_{2}$ are closed in $K$, and hence closed in $X$. Also, $K_{1} \cap K_{2}=\varnothing$ and $K_{1} \cup K_{2}=\bar{U} \cap X$, so that $\bar{K}_{1} \cup \bar{K}_{2}=\bar{U}$ and $\bar{K}_{1} \cap \bar{K}_{2}=\varnothing$. Since $p \in \overline{G \cap K} \subset \bar{K}_{1}$, it follows that $\bar{K}_{1}$ is a neighborhood of p. But $|f|=|g|$ on $G$, and hence on $\bar{K}_{1}$, and so $f=g=0$ on $\bar{K}_{1}$. Thus $f$ and $g$ belong to $\mathbf{J}_{p}$.

We have now proved that $(1) \Rightarrow(2)$ and that $(4) \Rightarrow(1) ;(2) \Rightarrow(3)$ is trivial. For (3) $\Rightarrow(4)$, note that $X$ is metrizable, separable and infinite, so that $\mathbf{C}_{0}(X)$ is infinite dimensional and separable. In particular, $\left|C_{0}(X)\right|=2^{\aleph_{0}}$, and so by $[9$, Théorème 
6.2] each nonclosed prime ideal of $\mathbf{C}_{0}(X)$ is the kernel of a discontinuous homomorphism; it is immediate that the same is true for a finite intersection of prime ideals.

Remarks. 1. The topological hypotheses on $X$ include the case where $X=X_{0} \backslash F$, $X_{0}$ compact and metrizable, $F$ a finite set of nonisolated points.

2. The metrizability of $X$ is heavily used in the proof, both for the details of the construction, and to ensure that arbitrary closed sets are zero-sets, though where possible the weaker supposition of second countability is explicitly used.

3. Recall that a point $p \in \beta X$ is called remote $[14,4.37]$ if $p \notin \bar{D}$ for any discrete set $D \subset X$. Thus if the set $W$ of isolated points has compact closure in $X$, then condition (1) of the theorem is just the statement that $p$ is remote. Note that with this condition on $W$ and assuming $\mathrm{CH}$ there are $2^{\mathrm{c}}$ remote points [14, Theorem 4.43].

Our argument shows in fact that, if $p$ is remote, then $\mathbf{J}_{p}$ is prime, but the converse to this is false. For let $X_{0}=\mathbf{N} \cup\{\infty\}$, and $X=\mathbf{N}$. Then each $\mathbf{J}_{p}$ is prime, but no $p \in \beta X \backslash X$ can be remote. Note that the result of [1] shows that in this case any homomorphism on $\mathbf{C}\left(X_{0}\right)$ is continuous on some finite intersection of the prime ideals $\left\{\mathbf{J}_{p}: p \in \beta X \backslash X\right\}$.

\section{REFERENCES}

1. W. G. Bade and P. C. Curtis, Jr., Homomorphisms of commutative Banach algebras, Amer. J. Math. 82 (1960), 589-608.

2. H. G. Dales, $A$ discontinuous homomorphism from $C(X)$, Amer. J. Math. 101 (1979), 647-734.

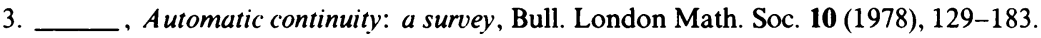

4. H. G. Dales and W. H. Woodin, An introduction to independence for analysts, London Math. Soc. Lecture Notes (to appear).

5. J. Esterle, Seminormes sur $C(K)$, Proc. London Math. Soc. 36 (1978), 27-45.

6. __ Solution d'un problème d'Ërdos, Gillman et Hendriksen et application a l'étude des homomorphisme de $C(K)$, Acta Math. Acad. Sci. Hungar. 30 (1977), 113-127.

7. Sur l'existence d'un homomorphisme discontinu de $C(K)$, Proc. London. Math. Soc. 36 (1978), 46-58.

8. Injection de semigroupes divisibles dans des algèbres de convolution et construction d'homomorphismes discontinus de $C(K)$, Proc. London Math. Soc. 36 (1978), 59-85.

9. Homomorphismes discontinus des algèbres de Banach commutatives séparables, Studia Math. 66 (1978), 119-141.

10. L. Gillman and M. Jerison, Rings of continuous functions, Springer-Verlag, New York, 1976.

11. S. Grabiner, A formal power series operational calculus for quasinilpotent operators. II, J. Math. Anal. Appl. 43 (1973), 170-192.

12. B. E. Johnson, Norming $C(\Omega)$ and related algebras, Trans. Amer. Math. Soc. 220 (1976), 37-58.

13. A. M. Sinclair, Homomorphisms from $C_{0}(\mathbf{R})$, Proc. London Math. Soc. 11 (1975), 165-174.

14. R. C. Walker, The Stone-Čech compactification, Springer-Verlag, Berlin, 1974.

15. W. H. Woodin, Set theory and discontinuous homomorphisms from Banach algebras, Mem. Amer. Math. Soc. (to appear).

School of Mathematics, University of Leeds, Leeds LS2 9JT, ENGLAND

Department of Mathematics, Faculty of Science, Australian National University, GPO Box 4, Canberra, ACT 2601, Australia 